\title{
The trajectory of a dot crossing a pattern of tilted lines is misperceived
}

\author{
ANNA LISA CESÀRO and TIZIANO AGOSTINI \\ University of Trieste, Trieste, Italy
}

\begin{abstract}
The straight trajectory of a dot crossing a pattern of tilted lines is perceived as being sinusoidal. Manipulation of the size of the angle between the trajectory and the tilted lines, the velocity of the dot, and the distance between the tilted lines shows that the magnitude of the illusion is inversely proportional to the size of the incidence angle, to velocity, and to the distance between the lines. The illusion is interpreted as being the result of an integration process of local distortions occurring at the intersections with the tilted lines.
\end{abstract}

In illusions of direction and shape, the direction or orientation of a contour is perceptually altered (Coren \& Girgus, 1978). A large number of optical geometrical illusions are examples of this kind of effect (Burmester, 1896; Hering, 1861; Jastrow, 1891, 1892; Lipps, 1897; Orbison, 1939a, 1939b; Zöllner, 1860). Figure I depicts the Hering variant of the Wundt-Hering illusion, in which two straight, horizontal lines are perceived to be curved. Another example is the Poggendorff illusion, in which two collinear portions of an oblique line appear to be misaligned, as shown in Figure 2 . When the oblique line is replaced by a translating dot, the illusion of nonalignment becomes even more pronounced (Nihei, 1973, 1975). Fineman and Melingonis (1977) independently investigated a kinetic Poggendorff illusion with similar results. Also, Wenderoth and Johnson (1983) reported for the kinetic Poggendorff illusion an effect that was larger than that for the static one.

Swanston (1984) studied the movement path distortion in other displays that produce illusions of line orientationthe Hering and the Zöllner figures. He intended to determine whether such dynamically generated subjective contours and constant real contours behave similarly. He reports that both the Hering and the Zöllner kinetic illusions reproduce the form of the static versions. He claims that an account may be given of these kinetic illusions that is similar to the account that has been made for the static case, in terms of the integration of small displacements occurring at each intersection.

In his work, Swanston (1984) also reports that a dot crossing a line that is inclined to its horizontal direction of movement appears to undergo a vertical displacement. This effect was previously observed by Nihei $(1973,1975)$ and by Wen-

This research was supported by CNR Grant 92.01065.PF67. We thank Lawrence Arend, Paolo Bozzi, Nicola Bruno, Fulvio Domini, Walter Gerbino, Maggie Shiffrar, lan Verstegen, and the perception group of our department for their valuable advice and critical comments. Correspondence concerning this article should be addressed to T. Agostini, Dipartimento di Psicologia, Università di Trieste, via dell'Università, 7, 34123 Trieste, Italy (e-mail: agostini@uts.univ.trieste.it). deroth and Johnson (1983). They reported that the dot appeared to move toward the nearest part of the line and then away again after the crossing, so that a noticeable "blip" was introduced into the movement. Whereas they just report informal observations, Swanston measured this effect. He determined that the change in the perceived path was a function of the orientation of the line. The form of this function is very similar to that reported by Carpenter and Blakemore (1973) for perceived angle expansion between static lines.

We observed a new kinetic illusion. When a dot translates along a straight horizontal path and intersects a pattern of tilted lines, its trajectory is perceived as being sinusoidal (see Figure 3 ; recently we named this new illusion the slalom effect [Cesàro \& Agostini, 1997]). This study has two main purposes. The first is to determine whether there is any similarity between the new effect and the kinetic effects reported in the literature. The second purpose is to test whether this illusion can be accounted for in terms of the integration of small displacements occurring at each intersection, as suggested by Swanston (1984) for the Hering and Zöllner illusions. When a dot translates along a horizontal path and intersects a line inclined to its direction, it appears to undergo a vertical displacement (Swanston, 1984). In our illusion, there is a sequence of tilted lines; thus, a sequence of displacements should occur. Nevertheless, in the trajectory of the dot, the sequence of displacements is not visible. The dot smoothly moves like a wave. This is consistent with an interpretation of the illusion in terms of the integration of the displacements occurring at each intersection.

\section{Overview of Experiments}

We performed three experiments. In the first experiment, we manipulated the angle between the trajectory of the moving dot and the tilted lines. The aim was to determine whether there was any change in the trajectory as a function of the angle. Since both static and kinetic illusions of direction are angle-dependent (Coren \& Girgus, 1978; Swanston, 1984), we expected that our illusion also would depend on the angle. We also expected the function to be similar to that reported in the literature for illusions of di- 


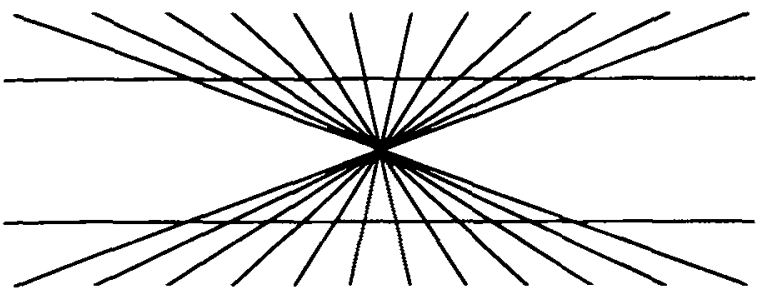

Figure 1. The Hering variant of the Wundt-Hering illusion. The two horizontal lines are drawn straight and parallel, but they are perceived to be curved.

rection. This means that, in the range we used $\left(30^{\circ}-60^{\circ}\right)$, the illusion should increase as the angle decreases.

In the second experiment, we varied both the incidence angle and the velocity of the dot. Nihei (1973) had found both an effect of velocity and a significant interaction between angle and velocity in the kinetic Poggendorff illusion. Swanston (1984) instead reports no velocity effect for the kinetic Zöllner illusion. Nihei (1973) used five velocities in the range of $2^{\circ}-45^{\circ} / \mathrm{sec}$, whereas Swanston used only two different velocities, $2 \% \mathrm{sec}$ and $4 \% \mathrm{sec}$. During preliminary observations, we noticed that there was an optimal range of velocities. Outside this range, it was difficult to follow the dot, either because it was too slow or because it was too fast. This range was $0.78^{\circ}-3 \% / \mathrm{sec}$, which is very similar to the one used by Swanston. According to Swanston's findings, within that range we should expect no effect of velocity on the magnitude of the illusion.

In the third experiment, we manipulated the distance between the tilted lines. This was done in order to test whether the density of the inducing field has an effect similar to that reported for other illusions. Furthermore, a direct relation between the magnitude of the illusion and the density of the inducing field would support an interpretation in terms of the integration of small displacements occurring at each intersection, as opposed to an interpretation in terms of just a sequence of displacements. If the integration occurs, manipulating the distance between the inducing lines should affect the magnitude of the illusion. In particular, the illusion should decrease as the distance between the lines is increased. It has been suggested, in fact, that the distorting effects of one line on another or on a movement path are localized (Carpenter \& Blakemore, 1973; Swanston, 1984). Manipulating the distance between the lines changes the rate of trajectory falling within the field of influence of the lines - that is to say, the rate of distorted trajectory. If no integration occurs, the rate of distorted trajectory is irrelevant, since the magnitude of each single distortion determines the magnitude of the illusion. But if the distortions are integrated across the whole trajectory, the rate of distorted trajectory is critical for the magnitude of the resulting illusion. Decreasing the distance between the intersections increases the rate of distorted trajectory; thus, we expect our illusion to increase when the distance between the tilted lines is decreased.

\section{EXPERIMENT 1}

This experiment was performed to verify (1) whether the size of the illusion is angle-dependent and (2) in case of an effect of the angle, whether the illusion is inversely proportional to the size of the angle.

\section{Method}

Observers. Ten students from the University of Trieste volunteered to serve as observers in this experiment. All had normal or corrected-to-normal vision. They were naive as to the purpose of the experiment.

Apparatus. The stimuli were displayed on the high-resolution RGB monitor ( $1,280 \times 1,024$ pixels, $120-\mathrm{Hz}$ refresh rate $)$ of a Silicon Graphics Indigo computer workstation.

Stimuli and Procedure. There were five different patterns, one for each of the selected incidence angles (see Figure 4a). The black solid lines $(0.27 \mathrm{~mm}$ thick) were displayed on a white background. The distance between the observer and the computer screen was $70 \mathrm{~cm}$. The size of the module used to generate each pattern is reported in Figure $4 \mathrm{~b}$. The module was repeated six and a half times, and the whole pattern was in the center of the screen. The black dot had a diameter of $0.54 \mathrm{~mm}$ and moved horizontally from left to right along a straight trajectory $(230 \mathrm{~mm}$; see the dashed lines of Figure $4 \mathrm{a})$ at a constant velocity of $19 \mathrm{~mm} / \mathrm{sec}\left(1.55^{\circ} / \mathrm{sec}\right)$. The display was seen through a circular black reduction screen (diameter, $220 \mathrm{~mm}$ ), which was centered with the center of the pattern.

At the beginning of each trial, the pattern of tilted lines was visible on the screen. The dot started moving behind the left side of the reduction screen and crossed the pattern of solid lines ending its trajectory behind the right side of the reduction screen. At this point, the whole display disappeared, and, $1 \mathrm{sec}$ later, an adjustable vertical bar $(0.27 \mathrm{~mm}$ thick) appeared $40 \mathrm{~mm}$ above the center of the screen. Using two keys of the keyboard, the observers had to adjust the length of the bar to make it equal to the amplitude of the perceived sinusoidal trajectory. The initial length of the bar was randomly assigned (range, 0.27 to $8 \mathrm{~mm}$ ) and varied with steps of $0.27 \mathrm{~mm}$. The observers could see the experimental display and correct the adjustment as many times as they wished. When they were satisfied, they moved to the next trial by pressing a key. There were 20 trials (five patterns presented four times), in a within-subjects design. The trials were randomized for each observer. Before the experimental session, the observers performed a short training task that was similar

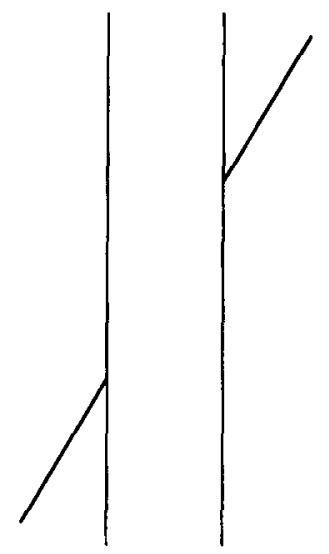

Figure 2. The Poggendorff illusion. The two parts of the oblique line are geometrically collinear, but they are perceived as misaligned. 


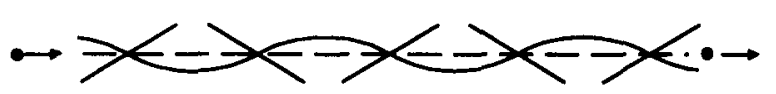

Figure 3. When a dot moving along a straight horizontal trajectory (dashed line) intersects a pattern of tilted lines, its trajectory is perceived as being sinusoidal (solid line).

to the experimental one. At the beginning of the training, the observers were asked to verbally describe the dot trajectory. This method was used in order to ensure that the observers spontaneously perceived a sinusoidal trajectory. The observers were tested individually. Viewing was binocular. The experimental room was dark during the experiment.

\section{Results and Discussion}

The results of Experiment 1 are shown in Figure 5. A one-way analysis of variance (ANOVA) revealed an effect of angle size $[F(4,36)=31.603, p<.001]$.

These results show that the illusion is angle-dependent, confirming our prediction. Furthermore, the magnitude of the illusion is inversely proportional to the size of the angle, following the same function as that reported by Swanston (1984) for the displacement in the trajectory of a dot crossing an oblique line. This kind of function is reported in the literature for both static and kinetic illusions of direction. The fact that these illusions behave very similarly as to the role played by the angle supports the idea that a common mechanism is involved.

\section{EXPERIMENT 2}

This experiment was performed to verify (1) whether the illusion is velocity-dependent and (2) whether there is any interaction between velocity and incidence angle.

\section{Method}

Observers. Ten students from the University of Trieste volunteered to serve as observers in this experiment. All had normal or corrected-to-normal vision. They were naive as to the purpose of the experiment.

Apparatus. The apparatus was the same as that in the first experiment.

Stimuli and Procedure. The nine stimuli used in this experiment resulted from the combination of three of the patterns used in Experiment $1\left(30^{\circ}, 45^{\circ}, 60^{\circ}\right)$ and three different velocities $-9.5 \mathrm{~mm} / \mathrm{sec}$, $19 \mathrm{~mm} / \mathrm{sec}$, and $38 \mathrm{~mm} / \mathrm{sec}(0.78 \% \mathrm{sec}, 1.55 \% \mathrm{sec}$, and $3.11 \% \mathrm{sec})$. All the features of the stimuli were the same as those in Experiment 1, except for the size of the reduction screen (diameter, $85 \mathrm{~mm}$ instead of $220 \mathrm{~mm}$ ), the position of the adjustable bar $(5.4 \mathrm{~mm}$ instead of $40 \mathrm{~mm}$ above the center of the screen), and its variation rate (steps of $0.027 \mathrm{~mm}$ instead of $0.27 \mathrm{~mm}$ ). The procedure was the same as that in Experiment 1, except for the number of trials, which was 36 (nine stimuli repeated four times).

\section{Results and Discussion}

The results of Experiment 2 are shown in Figure 6. A two-way ANOVA revealed a main effect of both angle size $[F(2,18)=97.016, p<.001]$ and velocity $[F(2,18)=10.274$, $p \leq .001]$. No significant interaction was found.

These results show that velocity also plays a role in the illusion, with no interaction with the angle. The illusion is stronger with the lowest velocity and weaker with the highest. Nihei (1973) reports, for the kinetic Poggendorff illusion, both an effect of velocity and a significant interaction with the angle. The shape of his functions is quite different from that of ours. But the range of velocities he used does not allow us to compare the data. In fact, only his lowest velocity falls in the range we used. On the other hand, Swanston (1984) reports no effect of velocity for the kinetic Zöllner illusion in a range of velocities very similar to the one we used, so it is reasonable to compare our findings with his. The difference between our findings and Swanston's findings could be due to several factors. It might be that velocity affects the two illusions in a different way; so an effect might be found in the kinetic Zöllner illusion with a different range of velocities. Alternatively, it could be that the kinetic Zöllner illusion is not affected at all by velocity, whereas our illusion is affected. In general, it seems that data on the role of velocity in kinetic illusions are contradictory and do not allow us to draw clear conclusions. The only thing we can say is that the effect of velocity seems to vary according to the range of velocities used.

\section{EXPERIMENT 3}

This experiment was performed to verify (1) whether the magnitude of the illusion depends on the distance between the inducing lines and (2) in case of an effect of the

a

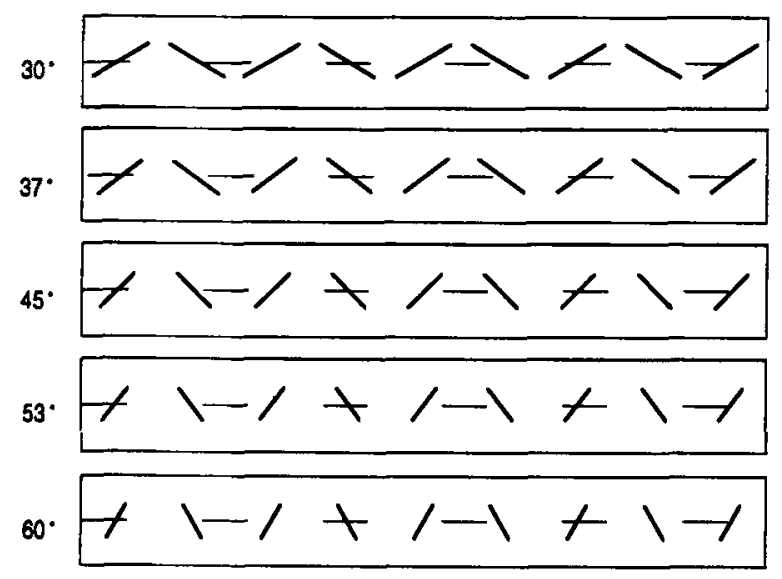

b

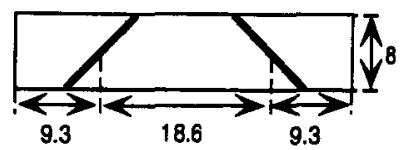

Figure 4. (a) The five patterns used in Experiment 1. The size of the incidence angle is reported on the left. The dashed lines represent the physical trajectory of the dot. (b) An example (45 $\left.{ }^{\circ}\right)$ of the modules that were used to generate the patterns. Sizes, reported in millimeters, are the same for all of the five patterns, whereas the angle between the tilted lines and the horizontal (the incidence angle) varies for each pattern. 


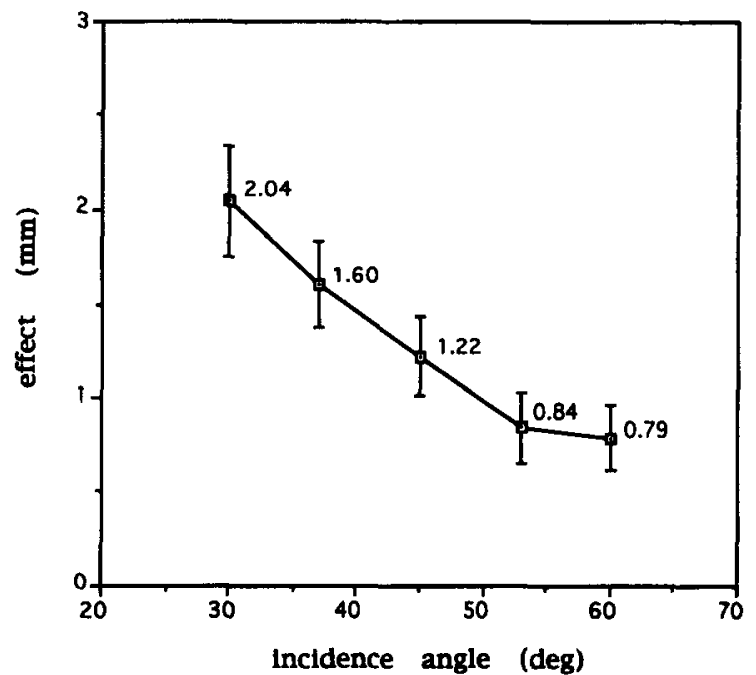

Figure 5. The data from Experiment 1. The amplitude of the perceived sinusoidal trajectory is plotted as a function of the angle of incidence. Error bars represent standard errors.

distance, whether the illusion decreases as the distance is increased.

\section{Method}

Observers. Ten students from the University of Trieste volunteered to serve as observers in this experiment. All had normal or corrected-to-normal vision. They were naive as to the purpose of the experiment.

Apparatus. The apparatus was the same as those in the first and second experiments.

Stimuli and Procedure. There were three different patterns. In each of them, the distance between the tilted lines was different: $13.3 \mathrm{~mm}, 18.6 \mathrm{~mm}$, and $23.9 \mathrm{~mm}$. In all of the three patterns, the incidence angle was $30^{\circ}$, and the velocity was $19 \mathrm{~mm} / \mathrm{sec}\left(1.55^{\circ} / \mathrm{sec}\right)$. All the features of the stimuli were the same as those in Experiment 2, except for the number of trials, which was 15 (three stimuli repeated five times). The procedure was the same as that in Experiments 1 and 2, except for the instructions. In this experiment, it was stressed that the judgment be given on the basis of the amplitude of the sinusoidal trajectory, regardless of its frequency.

\section{Results and Discussion}

The results of Experiment 3 are shown in Figure 7. A one-way ANOVA revealed an effect of the distance between the lines $[F(2,18)=12.745, p<.001]$.

These results show that the magnitude of the illusion depends on the distance between the inducing lines. When the lines are closer, the illusion is stronger. This confirms our predictions and supports an interpretation of the illusion as the integration of displacements occurring in the dot trajectory when crossing the tilted lines.

Furthermore, these results show another similarity to static illusions, in particular to the Zöllner illusion. In the Zöllner illusion, in fact, the density of intersecting lines affects the magnitude of the effect. The illusion is stronger when the density of the lines is higher--that is, when the lines are closer (Wallace \& Crampin, 1969).

\section{GENERAL DISCUSSION}

We observed a new kinetic illusion of direction and shape. A dot moving straight across a pattern of tilted lines is perceived to have a sinusoidal trajectory. The results of our experiments show that the magnitude of the illusion depends on the size of the angle of intersection, on the velocity of the dot, and on the distance between the inducing lines. All of the three parameters are related to the size of the illusion by an inverse relation.

These results show that this new illusion behaves very similarly to both the kinetic and the static illusions of direction that are reported in the literature. As far as the effect of the angle is concerned, the data reported in the literature are very coherent, showing that the maximum effect is at around $15^{\circ}$, decreasing slowly until $90^{\circ}$ and more rapidly until $0^{\circ}$. Our data are consistent with those findings, the size of our illusion being greater when the angle is $30^{\circ}$ and smaller when it is $60^{\circ}$.

Whereas the role of the angle in kinetic illusions is clear, the role of velocity is not. In fact, the data reported on the effect of velocity in some kinetic illusions are incomplete and seem contradictory. Thus, a more systematic study of how velocity influences the illusions is needed in order to draw conclusions on this aspect.

The effect of the density of the background pattern has not been studied as extensively as has the effect of the angle. Nevertheless, there are data showing for the static Zöllner illusion an effect similar to the one we report for our illusion. The fact that the illusion tends to be stronger when the density of the background is increased not only emphasizes the similarities between our illusion and those previously studied but also supports an interpretation in terms of the integration of small displacements occurring at each

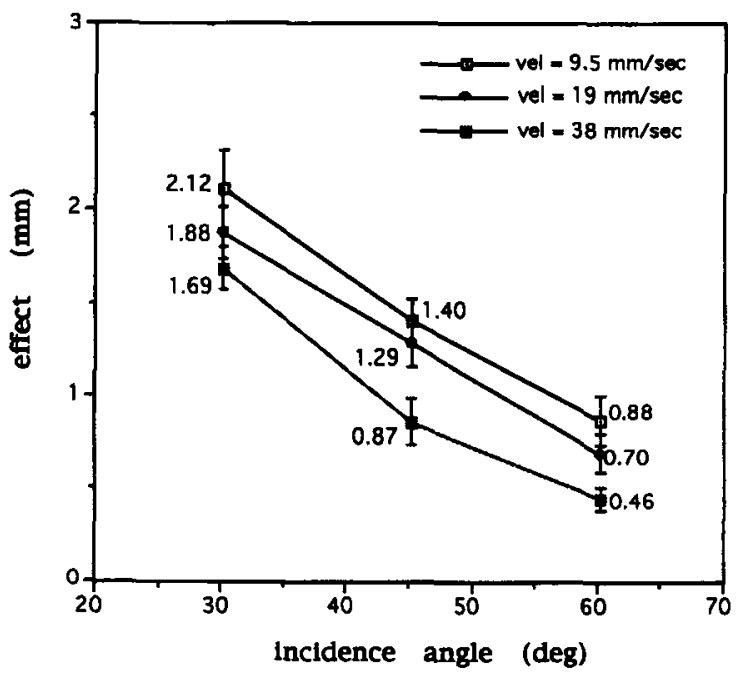

Figure 6. The data from Experiment 2. The amplitude of the perceived sinusoidal trajectory is plotted as a function of the angle of incidence for each of the three velocities. Error bars represent standard errors. 


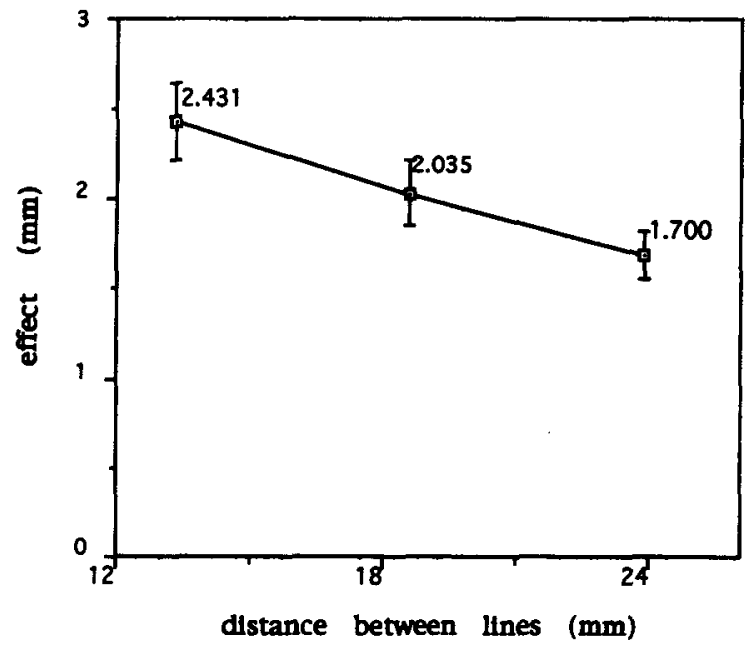

Figure 7. The data from Experiment 3. The amplitude of the perceived sinusoidal trajectory is plotted as a function of the distance between the tilted lines. Error bars represent standard errors.

intersection. In fact, as we pointed out in the overview of the experiments, if no integration occurred, the distance between the inducing lines should be irrelevant.

Since this new illusion has much in common with previously observed kinetic illusions, there are good reasons to believe that they all depend on a common mechanism. Moreover, kinetic illusions in general have features very similar to those of static ones. As suggested by Swanston (1984), the strong possibility is that there is a common mechanism underlying the interactions, on the one hand, between static orientations and, on the other, between movement directions. Some form of reciprocal inhibition has been suggested as the origin both of orientation effects (Carpenter \& Blakemore, 1973) and of successive movement repulsion effects (Levinson \& Sekuler, 1976). Swanston suggests a similar basis for the effects of the interaction between line orientation and movement direction. He claims either that there may be a link between the systems responsive to movement direction and to orientation, such that they exert reciprocal inhibition on each other's activity, or that both systems might feed into a higher level of analysis, in which static and dynamic direction is processed as a stimulus characteristic. Neurophysiological studies on the macaque middle temporal visual area have shown that there are neurons selectively responsive to a direction of motion and to a nearly perpendicular orientation (Albright, 1984). It is plausible that similar neurons exist also in the human visual cortex, and their activity could be related to the effects observed in the kinetic illusions. Locally, in fact, the distortion occurring in these illusions is the tendency of the dot to normalize with respect to the line orientation. So, the displacement in the dot trajectory that was measured by Swanston might be an instance of the activity of this kind of direction-orientation selective neurons.
So far, we have dealt with the local distortion in kinetic illusions. As we claimed before, the perceived sinusoidal trajectory in our illusion is probably due to a sort of integration of the distortions occurring locally at each linetrajectory intersection. When the dot approaches the line, its trajectory is distorted toward the normalization-that is, the dot bends to enter the line perpendicularly. We hypothesize that its tendency would be to continue along this virtual trajectory, but its physical trajectory is straight. The perceived trajectory would be the result of a compromise between the two trajectories.

If a similar process occurs, it is clear how the size of the local distortion affects the size of the illusion. This kind of process also explains the effect of the distance between the lines. When the lines are closer, the part of nondistorted trajectory between each pair of lines is very short, as compared to the distorted one. For this reason, the perceived trajectory is more distorted. On the contrary, when the lines are more separated, there is a larger part of nondistorted trajectory that causes the perceived trajectory to be less distorted.

We are aware that our explanation of the illusion is very tentative. Nevertheless, we believe that further investigation will be able to refine it. Moreover, we think that, as long as neurophysiological evidence cannot fully explain visual phenomena, it is important to carry out careful observations and psychophysical measures in order to consolidate our knowledge of these phenomena, beyond any explanation.

\section{REFERENCES}

ALBRIGHT, T. D. (1984). Direction and orientation selectivity of neurons in visual area MT of the macaque. Journal of Neurophysiology, 52 , $1106-1130$

BURMESTER, E. (1896). Beiträge zur experimentellen Bestimmung geometrisch-optischer Täuschungen [Contributions to the experimental determination of the optical-geometrical illusions]. Zeitschrift für Psychologie, 12, 355-394.

Carpenter, R. H. S., \& Blakemore, C. (1973). Interactions between orientations in human vision. Experimental Brain Research, 18, 287-303.

CEsÀro, A. L., \& Agostini, T. (1997). Misperceiving the trajectory of a moving dot: Further observations [Abstract]. Investigative Ophthalmology \& Visual Science, 38, S375.

Coren, S., \& Grrgus, J. S. (1978). Seeing is deceiving: The psychology of visual illusions. Hillsdale, NJ: Erlbaum.

Fineman, M. B., \& Melingonis, M. P. (1977). The effect of a moving dot transversal on the Poggendorff illusion. Perception \& Psychophysics, 21, 153-156.

Hering, E. (1861). Beiträge zur Physiologie [Contributions to physiology] (Vol. I). Leipzig: Engelman.

JASTROW, J. (1891). A study of Zoellner's figures and other related illusions. American Journal of Psychology, 4, 381-398.

Jastrow, J. (1892). On the judgement of angles and positions of lines. American Journal of Psychology, 5, 214-221.

Levinson, E., \& SeKULER, R. (1976). Adaptation alters perceived direction of motion. Vision Research, 16, 779-781.

LiPPS, T. (1897). Raumausthetik und geometrisch-optische Täuschungen [Space aesthetics and optical-geometrical illusions]. Leipzig: Barth. NiHE1, Y. (1973). A preliminary study on the geometrical illusion of motion path: The kinetic illusion. Tohoku Psychologica Folia, 32, 108-114.

NIHEI, Y. (1975). The effect of direction of motion on the magnitude of the geometrical illusion of motion path: The kinetic illusion (II). Tohoku Psychologica Folia, 34, 88-94. 
ORBISON, W. D. (1939a). The correction of an omission in "Shape as a function of the vector field." American Journal of Psychology, 52, 309. ORBISON, W. D. (1939b). Shape as a function of the vector field. American Journal of Psychology, 52, 31-45.

SWAnston, M. T. (1984). Displacement of the path of perceived movement by intersection with static contours. Perception \& Psychophysics, 36, 324-328.

Wallace, G. K., \& Crampin, D. J. (1969). The effect of background density on the Zöllner illusion. Vision Research, 9, 167-177.

Wenderoth, P., \& Johnson, M. (1983), Relationships between the kinetic, alternating-line, and Poggendorff illusions: The effects of inter- stimulus interval, inducing parallels, and fixation. Perception \& Psychophysics, 34, 273-279.

ZölLNER, F. (1860). Über eine neue Art von Pseudoskopie und ihre Beziehung zu den von Plateau und Oppel beschriebenen Bewegungsphänomenon [On a new kind of pseudoscopic viewing and its relationship with the motion-phenomenon described by Plateau and Oppel]. Annalen der Physik, 110, 500-525.

(Manuscript received January 19, 1996; revision accepted for publication April 23, 1997.) 